\title{
Author Index to Volume 88
}

\section{$\mathbf{A}$}

Abbasi, Ibrahim 344

Abdullayev, Rakif 929

Abdul Razak, Muhammad Aliff 888

Abeles, Shira R. 1130

Abiola, Annie 1124

Abong'o, Daisy 301

Abrahão, Jônatas 1180

Abu-Madi, Marawan A. 1185

Accrombessi, Manfred M. K. 292

Achan, Jane 744

Achilla, Rachel 940

Acosta, Héctor 472

Adamani, William 441

Adams, A. Paige 455

Adaui, Vanessa 774

Afrane, Yaw A. 29

Agampodi, Suneth B. 184

Agbenyega, Tsiri E. 414

Agonafir, Mulualem 785

Agudelo-Flórez, Piedad 178

Aguirre, Hernán D. 407

Agunbiade, Abdulkareem 191

Ahmed, Dilruba 661

Ahmed, Kwaku 1028

Ahmed, Rafi 172

Aina, Oluwagbemiga 14

Akhtar, Sania 519

Akindele, Samuel 14

Akintonwa, Alade 14

Akinyede, Akinwumi 14

Akondy, Rama S. 172

Akram, Muhammad 519

Alam, Md Tauqeer 850

Alba, Milena 774

Alborzi, Abdolvahab 868

Alducin, César 566

Alexander, Neal 254

Ali, Abdullah Suleiman 289

Ali, Ibrahim 785

Ali, Said M. 144, 626

Alifrangis, Michael 536, 1124

Allonso, Diego 506

Alphonsus, Kal 441

Alpuche-Aranda, Celia 446

Alto, Barry W. 497

Alvarado-Otegui, Julián A. 872

Alvarez, Danilo 116

Ame, Shaali M. 626

Amidou, Samie 390

Amirova, Kamala 929

Amnuaysirikul, Jack 1138

Amoah, Abena S. 1028

Amor, Aranzazu 43

Amour, Caroline 390

Amran, Fairuz 704

Amza, Abdou 138

Anderson, Donald M. 908

Anderson, Jennifer M. 583

Andrews, Jason R. 626

Andriamandimby, Soa Fy 329

Andriantsoanirina, Valérie 37

Angelakis, Emmanouil 765

Angeletti, Davide 733

Anishchenko, Michael 1159

Apiwathnasorn, Chamnarn 254

Appawu, Maxwell 309

Arana-Guajardo, Ana 153

Arango, Eliana 1093
Araujo, Constance A. A. 592

Araujo, Nicole A. A. 592

Araujo, Sonia M. H. A. 592

Arens, Theo 5

Arevalo, Jorge 774

Argüello, D. Fermín 997

Arinaitwe, Emmanuel 736

Armignacco, Orlando 364

Armijos, M. Veronica 689

Armstrong, Margaret 1032

Aryeetey, Yvonne A. 1028

Asad, Saba 519

Asoala, Victor 309

Atiku, Linda A. 381

Awodele, Olufunsho 14

Aydin-Schmidt, Berit 289

Ayers, Tracy 132, 267

\section{B}

Babji, Sudhir 390

Bailey, Robin L. 138

Baltzell, Kimberly A. 289

Banegas, Engels Ilich 850

Banks, Ian 1070

Bannister-Tyrrell, Melanie 65

Baoliang, Xu 770

Bara, Mohamed Ould 946

Barbiéri, Clara Lúcia 559

Barrett, Alan 172

Barros, Elvino José Guardão 54

Barry, Alyssa E. 718

Bartos, Alisa J. 1152

Bassene, Hubert 339

Bastiaens, Guido J. H. 5

Bauer, Kristen 73

Bausch, Daniel G. 241

Bautista, Christian T. 929

Bayoh, M. Nabie 301

Beare, Nicholas A. V. 542

Beaty, Barry J. 260

Becker, Ingeborg 153

Behnke, Jerzy M. 1185

Beltrán, Manuela 997

Benacer, Douadi 704

Benard, Gil 236

Benedet, Christophe 37

Benito, Agustín 43, 1087

Benjamin, Seleena 490

Bennett, Andrew J. 241

Benson, Susan 481

Berger, Luise 198

Bergquist, Robert 409

Bergren, Nicholas A. 1170

Berl, Erica 103, 1159

Berman, Jonathan 216

Berzosa, Pedro 43, 1087

Besnard, Lydia 329

Bessong, Pascal 390

Betoulle, Jean-Luc 116

Bettinardi, David 497

Bhattarai, Rachana 1011

Bhutta, Zulfiqar 390

Bichha, Ram Padarath 464

Bickersmith, Sara A. 319

Biggerstaff, Brad J. 464

Bigira, Victor 736

Bijker, Else M. 5
Bikindou, Alain 982

Billingsley, Peter F. 5

Binder, Sue 426

Birbeck, Gretchen L. 315, 542

Bisanzio, Donal 48

Bishop, Henry 1062

Bishop, Henry S. 601

Bi, Yan 526

Björkman, Anders 289

Blacksell, Stuart D. 932

Black, William C. 381

Blair, Carol D. 260

Blanton, Curtis 645

Blevins, Tia M. 1159

Bligh, John 1032

Blyth, Christopher C. 481

Boakye, Daniel 309

Boakye, Daniel A. 1028

Bodeau-Livinec, Florence 292

Boegler, Karen A. 381

Boggild, Andrea K. 774

Bogoch, Isaac I. 626

Bojdi, Amin 469

Bokhari, Habib 519

Bomblies, Arne 727

Bonaparte, Matthew I. 962

Bond, Nell 241

Borchert, Jeff N. 381

Bosco-Lauth, Angela 1159

Botto-Mahan, Carezza 285

Bouchier, Christiane 37

Boudreaux, Carole 1062

Boué, Franck 795

Boufana, Belgees 795

Boutellis, Amina 1180

Bradley, Kristy K. 157

Brault, Aaron C. 1159

Bravo, Francisco 1207

Breiman, Robert F. 513

Brey, Paul T. 1070

Brito, Cristiana F. A. 325

Broncano, Nely 1041

Brooker, Simon J. 1102

Brown, Michael 1032

Brunette, Gary W. 376

Bucheli, Sandra Tamara Mancero 850

Bu-Coifiu-Fanego, Raisa 230

Budke, Christine M. 1011

Bui, Duy M. 472

Bulimo, Wallace 940

Burgos, Joaquín 1196

Burke, Rebecca 127

Burkhalter, Kristen 986

Burrus, Roxanne G. 319

Burt, David 167

Bustamante, Beatriz 1207

Bustamante, Dulce M. 630, 638

Bustinduy, Amaya L. 433

Byarugaba, Justus 733

Bygbjerg, Ib C. 536

\section{C}

Caccone, Adalgisa 897

Cajal, Silvana P. 578

Calderón, Maritza 552

Callender, Kevin 897

Calzada, Jose E. 572 
Camacho-Ortiz, Adrian 153

Campbell, Carl 426

Campos, Fernanda M. F. 325

Campos, Ricardo 285

Cançado, Guilherme Grossi Lopes 575

Canier, Lydie 37

Cantey, Paul T. 601

Cao, Hai-Xia 510

Carabin, Hélène 1011

Cardeño, John 1009

Cardinale, Eric 329

Cardinal, M. Victoria 872

Caridha, Diana 1138

Carletti, Isabelle 73

Carmona-Fonseca, Jaime 1093

Carpenter, L. Rand 162

Carriazo, Carlota S. 1146

Carrington, Lauren B. 689

Carroll, Darin S. 982

Carvalho, Luzia H. 325

Castelli, Francesco 397

Castillo, Gabriela 578

Castillo, Rosa 552

Cataño, Juan 1009

Cataño, Juan C. 407

Caterini, Luciano 364

Cerritos, René 186

Céspedes, Manuel 552

Cetron, Martin 172

Chakraborty, Subhankari Prasad 828

Chakravarty, Sumana 5

Chanampa, Mariana 578

Chang, Alicia H. 191

Chang, Kyu-Sik 24

Chansamouth, Vilada 932

Chao, Jiang 770

Charimari, Lincoln 645

Charlebois, Edwin 744

Charle, Pilar 43, 1087

Charlwood, Derek 536

Charnetzky, Dawn 986

Chaurasia, Ashish 1

Chaves, Luis F. 572

Chen, Xiaoguang 420

Chen, Xingwang 795

Cheng, Allen C. 411

Cheong, Fei Wen 835

Chico, Martha 1041

Chiodini, Peter L. 1032

Chirundu, Daniel 645

Chochlakis, Dimosthenis 698

Chong, Chee-Seng 888

Chundu, Kote 601

Chuquiyauri, Raul 1130

Cian, Amandine 1203

Cisse, Moumine 583

Clapasson, Andrea 211

Clark, Tamara D. 744

Clarke, Carmen F. 157

Cleaveland, Sarah 513

Clemens, John 144

Coffey, Michelle 986

Cohen, Adam L. 132

Colley, Daniel G. 426

Collins, William 726

Colton, Leah 986

Conn, Jan E. 319, 1079

Cook, Robert L. 908

Cookson, Susan T. 645

Cooper, Philip J. 1041

Cordón-Rosales, Celia 116

Coronado, Ximena 285

Costa, Federico 359

Cot, Michel 292
Craig, Philip 795

Cravo, Pedro 43

Crespo, Manuel 1196

Cserti-Gazdewich, Christine M. 747

Cuello-Pérez, Margarett 178

Cui, Feng 510

Curran, Adrián 1196

Currie, Bart J. 411

Curtis, Kurt C. 1035

Cutler, Sally J. 513

\section{D}

D'Alessandro, Umberto 608

da Silva Junior, Geraldo Bezerra 54

Dabboussi, Fouad 1203

Dadzie, Samuel 309

Daher, Elizabeth De Francesco 54

Daher, Elizabeth F. 592

Damon, Inger K. 982

Dance, David A. B. 411

Das, Pradeep 222

Das, Sabyasachi 828

Das, Sumon K. 661

Das, Sushmita 222

Das, Vidya Nand Rabi 222

Ddungu, Henry 747

de Carvalho Sanches, Osimar 596

de Fátima Duarte, Eliane 670

De La Barrera, Rafael 73

de la Cruz-Hernández, Sergio I. 446

De La Torre, Patricia 275

de Lucio, Aida 1087

de Macedo, Antonio Vaz 575

de Mendoza, Carmen 789

de Oca, Griselda Montes 186

Deardorff, Eleanor R. 1159

Deen, Jacqueline 144

Dei-Cas, Eduardo 1203

del Angel, Rosa M. 446

del Giudice, Pascal 807

del Rio, Carlos 172

Delaunay, Pascal 807

Delbac, Frederic 1203

Delhaes, Laurence 1203

Dellagi, Koussay 329

Delorey, Mark 986

den Hartog, Julia 167

Desai, Meghna 301

Desai, Sarika 645

Desale, Adinew 785

Deshpande, Bhushan R. 376

Dessy, Francis 73

Desta, Kassu 785

Dhabangi, Aggrey 747

Dia, Ndongo 946

Diatta, Georges 339

Diawara, Aïssatou 1052

Diaz, Cesar 850

Dickey, Mary 1011

Dieng, Yémou 1124

Ding, Shu-Jun 510

Dion, Kirstin 897

Diop, Ousmane M. 946

Dixon, Kandice 778

Doherty, Tom 1032

Doiphode, Sanjay H. 1185

Dong, Xingqi 923

Dorn, Patricia L. 638

Dorsey, Grant 736, 744, 855

Dorta-Contreras, Alberto Juan 230

Doumbia, Seydou 583
Du, Jenny N. T. 191

Duarte, Elisabeth Carmen 670

Duarte, Pastora M. A. 592

Dumre, Shyam P. 677

Dunn, John R. 162

Durand, Rémy 37

Dyer, John R. 701

Dzik, Walter H. 747

Ebel, Gregory D. 1159

Eberhard, Mark E. 1062

Eberhard, Mark L. 601

Eckels, Kenneth H. 73

Eckhoff, Philip 817

Edupuganti, Srilatha 172

Eidex, Rachel B. 172

Eigege, Abel 441

Eisen, Damon P. 20

Eisen, Lars 260

Eisen, Rebecca J. 103, 381

El Alaoui, Hicham 1203

El Safadi, Dima 1203

Eldin, Carole 765

Elfving, Kristina 289

Elias, Susan P. 95

Ellis, William 216

Emerson, Ginny L. 982

Enriquez, Gustavo F. 872

Epstein, Judith E. 3

Erko, Berhanu 426

Esch, Gerald W. 1209

Esmael, Ahmed 785

Espasa, Mateu 914

Espinoza, Bertha 275

Essien-Baidoo, Samuel 1028

Eursitthichai, Veerachai 677

Evans, Darin S. 441

Eynde, Eva Van den 1196

Ezenwa, Vanessa O. 986

$\mathbf{F}$

Fairley, Jessica K. 48

Faith, Sitnah Hamidah 132

Falcó, Vicenç 1196

Falconar, Andrew K. I. 178

Faria, Flavia Helena Fidelis 575

Faruque, A. S. G. 661

Faye, Babacar 1124

Faye, Ousmane 583

Feikin, Daniel R. 513

Feiz-Erfan, Iman 601

Feldlaufer, Mark F. 619

Felzemburgh, Ridalva D. M. 359

Fenollar, Florence 339

Fernández, Cintia J. 893

Fernández-Martínez, Amalia 43

Fernandez, Roberto 319

Fernandez, Stefan 73, 677

Ferri, Fabrizio 364

Filler, Scott 855

Finkmoore, Brooke 359

Fisa, Roser 586

Fischer, Marc 464

Fischer, Peter U. 1035

Flexman, James 481

Flores-Aguilar, Hilario 446

Folk, Scott M. 1035 
Fong, Mun Yik 835, 883

Fontaine, Robert E. 923

Font, Bernat 914

Fontecha, Gustavo A. 850

Fontes, Cor J. F. 325

Foote, Eric M. 132, 757

Foray, Coralie 329

Foss, Kimberly 95

Franco, Marília Masello Junqueira 596

Freedman, David O. 397

Freitas, Guilherme Gomes 575

Freitas, Renata N. 937

Frobish, Daniel J. 862

Fujii, Yoshito 367

Funnell, Simon G. P. 411

\section{G}

Gaayeb, Lobna 1203

Gachara, George 940

Gage, Kenneth L. 381

Galarza, Ivonne E. 472

Galloway, Renee L. 704

Galvão, Márcio A. M. 937

Gao, Xiugong 1138

Garcia, Beatríz A. 893, 1146

García, Enid J. 997

García-Gubern, Carlos 472

Garg, Pankaj Kumar 1

Garlapati, Rajesh 778

Garnica, Roberto 630

Gaye, Oumar 1124

Gaynor, Bruce D. 138

Gendernalik, Alex 778

Gerez de Burgos, Nelia M. 1146

Gershman, Mark D. 954

Ghadimi Moghadam, Abdolkarim 868

Ghimire, Prakash 677

Giaya, Kris 89

Gibbons, Robert V. 73, 464, 677

Gieraltowski, Laura 132

Gil, José F. 578

Gilbert, Gwendolyn L. 1048

Gilman, Robert H. 552

Gimnig, John E. 301

Githeko, Andrew K. 29

Glass, Gregory E. 727

Glover, Simon J. 542

Glowicz, Janet 157

Godoy, Adrian 897

Godsey Jr., Marvin S. 986

Goh, Wai-Keong 20

Goldman, Ira 850

Gómez-Camarasa, Cristina 1003

Gomez-Morales, Maria Angeles 364

González, Enrique 186

González-Martín, Julián 914

González-Mateos, Silvia 446

Goshit, Danjuma 441

Gottlieb, Michael 390

Gotuzzo, Eduardo 552, 1212

Graham, Alan C. 103

Graham, Christine B. 381

Grams, Hans Rudi 677

Grattan, Lynn M. 908

Gratz, Jean 390

Greenhouse, Bryan 289

Gregory, Christopher J. 472

Grogl, Max 216, 1138

Grubaugh, Nathan D. 245

Gudiño, Marco 186

Guerra, Humberto 552
Guerrant, Richard L. 390

Gunasekera, Anusha 5

Guo, Yayi 651

Guo, Yuming 526

Guptill, Stephen C. 986

Gürtler, Ricardo E. 872

Gutiérrez, Maite 789

Guzmán, Maria G. 506

\section{H}

Haeupler, Alexandra 198

Hagan, José E. 359

Hagembe, Juliana 547

Hallett, Rachel 1124

Halliday, Jo E. B. 513

Halstead, Scott B. 212

Hamburger, Joseph 344

Hamel, Mary J. 301

Hamze, Monzer 1203

Handzel, Thomas 645

Han, Pauline 954

Han, Pauline V. 397

Hansson, Helle H. 536

Haque, Rashidul 390

Haque, Ubydul 727

Haque, Waziul 727

Harder, Yves 198

Harley, David 65

Harvey, Steven A. 1109

Hashizume, Masahiro 727

Hati, Amiya Kumar 828

Havlir, Diane 744

$\mathrm{He}$, Gao 923

He, Zeyuan 923

Hepburn, Matthew 929

Héraud, Jean-Michel 329

Hermsen, Cornelus C. 5

Hernández, Eric 186

Hernández, Jorge 566

Herrero, María D. 789

Hickman, Mark 216, 1138

Higa, Yukiko 681

Higgs, Stephen 1163

Hildreth, Stephen W. 962

Hill, Nigel 1070

Hills, Susan L. 464

Hiscox, Alexandra 1070

Hoang, Nguyen Vet 681

Hobohm, Dan 601

Hoekstra, Robert M. 267

Hoenig, Donald E. 95

Hoffman, Stephen L. 5

Hofmeister, Erik K. 1152

Homsy, Jaco 736

Hoshino, Keita 681

Hoyos, Carlos L. 578

Hsiang, Michelle 289

Hubiche, Thomas 807

Hu, Branda T. 962

Hu, JunJie 1209

Hu, Wenbiao 526

Hudson, Thomas 216

Hughes, James M. 132

Hunsperger, Elizabeth 472, 997

Idoko, Olubukola T. 414

Iglesias-González, Ivonne 230

Ikadai, Hiromi 367
Ikegaya, Satoshi 1217

Ikilezi, Gloria 744

Inglis, Timothy J. J. 614

Innis, Bruce L. 73

Ioannou, Ioannis 698

Iraola, Gregorio 1146

Isawa, Haruhiko 681

Ishida, Hiroshi 1138

Ismayilova, Rita 929

Iwamoto, Maya 367

Iwasaki, Hiromichi 1217

J

Jabbarova, Latafat 929

Jacups, Susan P. 490

Jagannathan, Prasanna 736

Jahanov, Musa 929

Jaichapor, Boonsong 245

James, Anthony A. 420

James, Eric R. 5

Janusz, Kristen B. 464

Jarvis, Joseph 1032

Jenkins, David 795

Jensenius, Mogens 397

Jentes, Emily S. 376, 954

Jeoffreys, Neisha 1048

Jiddawi, Mohamed 144

Jiménez-Valera, María 1003

Jing, Wang 770

Johnson, Barbara W. 116

Johnson, Jacob 216

Johnson, Petrina H. 490

Juarez, Marisa 578

Juliano, Jonathan J. 1116

Juneja, Surender 20

Junghae, Muthoni 513

Juris, Sherrie A. 95

\section{$\mathbf{K}$}

Kaatano, Godfrey M. 841

Kabatereine, Narcis B. 426

Kabir, M. Moktadir 727

Kabir, Mamun 390

Kading, Rebekah C. 108

Kadri, Boubacar 138

Kakuru, Abel 736, 744

Kaljee, Linda M. 144

Kampondeni, Sam D. 542

Kamya, Moses 855

Kamya, Moses R. 736, 744, 1102

Kanchana, Sawan 461

Kanchana, Supannakhon 461

Kang, Gagandeep 390

Kano, Flora S. 325

Kaplan, Ray M. 1052

Karanja, Diana M. S. 426

Karem, Kevin L. 982

Karhemere, Stomy 982

Kariuki, Curtis 344

Kariuki, Simon 301

Kaul, Surinder 1070

Kaye, Angela 1070

KC, Khagendra 677

Keenan, Jeremy D. 138

Keiser, Jennifer 626

Keita, Alpha Kabinet 339

Keita, Somita 583

Kemere, Jordan 289 
Kengluecha, Ampornpan 245

Kesper, Norival 559

Keyserling, Harry 172

Khammanithong, Phasouk 1070

Khanam, Farhana 661

Kharabora, Oksana 1116

Khim, Nimol 37

Khosravi, Nasrin 469

Kiihl, Samara 651

Killeen, Gerry F. 809

Kilpatrick, A. Marm 116

Kim, Heung Chul 24

Kim, Hyun Kyung 24

Kim, Ke Chung 1007

King, Charles H. 48, 344, 426, 433

King, Christopher L. 48

King, Jonathan D. 441

King, Raymond J. 986

Kiniboro, Benson 718

Kinung'hi, Safari M. 841

Kinsey, John 1163

Kironde, Fred 733

Kishamawe, Coleman 841

Kiszewski, Anthony 309

Kiszewski, Anthony E. 897

Kitron, Uriel 48, 433, 872

Kiwanuka, Noah 1102

Kiwuwa, Mpungu Steven 733

Kleinschmidt, Immo 897

Knobel, Darryn L. 513

Knoll, Elisabeth 319

Ko, Albert I. 359

Kobayashi, Mutsuo 681

Kochhar, Sonali 414

Koivogui, Lamine 946

Komar, Nicholas 108, 116

Koop, Klaas 607

Koram, Kwadwo 309

Kosek, Margaret 390

Kosgei, Jackline 301

Koura, Ghislain K. 292

Krolewiecki, Alejandro J. 578

Kubrusly, Marcos 592

Kudzala, Amose 267

Kumar, Ashish 222

Kuwata, Ryusei 681

\section{$\mathbf{L}$}

Labruna, Marcelo B. 937

Lacerda, Marcus V. G. 325

Laclette, Juan Pedro 275

Ladrón de Guevara, Concepción 789

Lago, Mar 789

Lagunas, Mariana S. 1146

Lahmar, Samia 795

Lai, Yee-Ling 1065

Lal, Sekhar Chandra 222

Lambrechts, Louis 689

Lancaster, Mary J. 162

Lanciotti, Robert 172

Lanciotti, Robert S. 116

Lang, Dennis 390

Lara, Gustavo Henrique Batista 596

Larbi, Irene A. 1028

LaRocque, Regina C. 376, 954

Lau, Yee Ling 835, 883

Laver, Susan 645

Lavoy, Geoff 855

Lazo, John S. 216

Leder, Karin 397

Lee, Caleb 888
Lee, Dong-Kyu 24

Lee, Gwenyth 390

Lee, Hyun-Ho 24, 245

Lee, Rogan 1048

Lee, Sue J. 932

Leelayoova, Saovanee 352

Leite, Clarice Queico Fujimura 596

Lenhart, Audrey 254

Leontsini, Elli 1109

Lescano, Andres G. 319

Leung, Daniel T. 661

Levett, Paul N. 178

Ley, Benedikt 144

Li, Chenny 1065

Li, Furong 923

Li, Huan-Ying 918

Li, Juan 420

Li, Qigui 1138

Li, Yu 982

Li, Zhongjie 923

Libraty, Daniel H. 89

Lichtenwalner, Anne 95

Lietman, Thomas M. 138

Liew, Christina 888

Lima, Aldo 390

Limmathurotsakul, Direk 411

Lin, Jessica T. 1116

Lindesay, Janette 65

Lindoso, José Angelo Lauletta 559

Lindsay, Steven W. 1070

Lipman, Harvey 172

Liu, Jian 918

Liu, Lijuan 770

Llanos-Cuentas, Alejandro 774

Lo, Ami Colle 1124

Loan, Do Phuong 681

Lobo, Neil F. 301

Locatelli, Fabricio M. 578

Logan, Sarah 1032

Loharikar, Anagha 267

Long, Lewis S. 245

López, María R. 116

Lopez, Ruth 566

López-Martinez, Irma 446

Lorenzi, Olga D. 472

Loutan, Louis 397

Lowe, Rachel 971

Lubelczyk, Charles 95, 1159

Luce, Richard R. 997

Lucero, David E. 630

Ludert, Juan E. 446

Luo, Huiming 923

Luo, Ping 962

\section{M}

Mabey, David C. W. 138

MacDonald, Laura 651

MacDonald, Luke 651

MacMillan, Katherine 103

Maco, Vicente 1212

Maco, Vicente P. 1212

Madejón, Antonio 789

Madsen, Laura B. 536

Maestre, Amanda 1093

Mafra, Cláudio L. 937

Magesa, Stephen M. 841

Magill, Alan J. 1138

Magnarelli, Louis A. 1159

Mahmud, Rohela 835

Maina, Alice N. 513

Majanja, Janet 940
Majwala, Albert 127

Malek, M. A. 661

Malhotra, Indu 48

Malima, Robert C. 841

Mallat, Hassan 1203

Mangia, Simone Henriques 596

Manitta, Joseph 20

Mannor, Kara M. 315

Manxia, Hu 770

Manyazewal, Tsegahun 547

Marcos, Luis A. 1035

Marinucci, Francesco 547

Mårtensson, Andreas 289

Martínez-Hernández, Fernando 275

Martínez-Ibarra, Jose Alejandro 275

Martinho, Anna Paula Vitirito 596

Marwanga, Doris 301

Mashauri, Fabian M. 841

Mason, Carl 390

Massone, Cesare 211

Massougbodji, Achille 292

Masuku, Humphreys D. 267

Mathenge, Penninah 645

Mathison, Blaine 1062

Mathison, Blaine A. 601

Matias, Abrahan 897

Matthias, Michael A. 184, 552

Maves, Ryan C. 552

Maxwell, Ellen 20

Mbaye, Maimouna Ndour 608

Mboera, Leonard E. G. 841

McCall, Philip J. 254

McCollum, Andrea M. 982

McElroy, Kristina 162

McKeon, Sascha N. 1079

McMenamy, Scott S. 245

McNair, James N. 862

McQuiston, Jennifer 162

Mduma, Estomih 390

Mediannikov, Oleg 339

Medina-Moreno, Sandra 547

Megid, Jane 596

Mejia, Rojelio 1041

Mejia Torres, Rosa Elena 850

Melanson, Vanessa R. 245

Meléndez, Paolo 552

Meloni, Dionigia 1203

Ménard, Didier 37

Mendoza, Meisy 850

Menon, Manoj 267

Mercer, Geoff 65

Mero, Chacha N. 841

Midzi, Stanley 645

Milagres, Bruno S. 937

Milheim, Lesley E. 986

Miller, Mark 390

Ming, Li 770

Miri, Emmanual S. 441

Mitra, Dipak 727

Miyamoto, Denise 236

Mnapo, Bridget 607

Mody, Rupal 929

Mohana-Borges, Ronaldo 506

Mohd Zain, Siti Nursheena 704

Molina, Israel 1196

Mombouli, Jean V. 982

Monroy, M. Carlota 638

Monroy, Maria Carlota 630

Montandon, Carlos E. 937

Monteiro, Maria de Lourdes 946

Monteon, Victor 566

Moore, Christopher C. 127

Moore, Elinor 1032

Moore, Sean M. 381 
Moraes, Giselle Hentzy 670

Morales-Betoulle, Maria E. 116

Morales-Betoulle, Maria Eugenia 108

Morán, Patricia 186

Moreno, Angelo C. 184

Moreno, Marta 319

Morof, Diane 645

Morris Jr., J. Glenn 908

Morrissey, Leslie A. 630

Moses, Cynthia 982

Moses, Lina M. 241

Mosites, Emily 162

Mostafavi, Irandokht 469

Mpanga, Joseph T. 381

Mpimbaza, Arthur 747

Msellem, Mwinyi 289

Muchiri, Eric 48, 344

Muchiri, Eric M. 433

Mueller, Ivo 718

Muhindo, Mary 736

Mukunzi, Silvanos 940

Mula, Patricia 43

Müller, María L. 116

Mulligan, Mark J. 172

Mungai, Peter 48, 344

Mungai, Peter L. 433

Mungthin, Mathirut 352

Muñoz-Garza, Fania Z. 153

Muñoz-Jordan, Jorge L. 997

Muñoz, Jorge 472

Muntau, Birgit 198

Musoke, Charles 747

Mutalib, Adeliza 1065

Mutebi, John-Paul 95, 103, 1159

Muth, Sinuon 1116

Mutuku, Francis 433

Muyembe, Jean J. 982

Muzoora, Conrad 127

Mwanga, Joseph R. 841

Mwangi, Josephat 940

Mzungu, Elton 344

\section{$\mathbf{N}$}

N'Goran, Eliézer K. 426

Naafs, Bernard 236

Naaglor, Tawee 352

Na-Bangchang, Kesara 677

Nabukeera-Barungi, Nicolette 747

Naderi, HamidReza 469

Nakiboneka-Ssenabulya, Deborah 747

Nale, Joe 718

Nankabirwa, Joaniter 1102

Nasirova, Emilya 929

Nasr, Sussann 855

Nasser, Julio R. 578

Nassirou, Baido 138

Navarro, Jordi 1196

Navarro, Marta 914

Navarro-Marí, José María 1003

Navegantes de Araújo, Wildo 359

Ncogo, Policarpo 43

Ndiaye, Daouda 1124

Ndiaye, Jean Louis 1124

Ndiaye, Magatte 1124

Ndiaye, Mbayame Niang 946

Ndimubanzi, Patrick C. 1011

Nebbia, Gaia 1032

Negri, Vanesa 578

Newton, Paul N. 932

Ng, Lee-Ching 888, 1065

Nga, Phan Thi 681
Ngo, Tue H. 162

Nguyen, Hai 1011

Nieves, Miriam 186

Nisalak, Ananda 677

Njenga, M. Kariuki 513

Njiri, James 940

Njoroge, Rose N. 940

Nnko, Soori E. 841

Nobre, Vandack 575

Nofchissey, Robert A. 1159

Noman, Nawajish 727

Noor, Ramadhani A. 608

Norris, Douglas E. 710

Norris, Laura C. 710

Nseng Nchama, Gloria 1087

Nunes, Marcio R. 455

Nunzi, Enrico 211

Nutman, Thomas B. 1041

\section{$\mathbf{O}$}

O’Guinn, Monica L. 245

O'Neil, Michael T. 1138

Obeng, Benedicta B. 1028

Oberste, M. Steven 472

Ocampo-Candiani, Jorge 153

Ocaña, Inma 1196

Ochiai, Leon 144

Ogah, Gladys 441

Ogola, Eric 513

Ogutu, Bernhards 414

Ohrt, Colin 1138

Okany, Charles 14

Olang, George 301

Olayemi, Sunday 14

Oliveira, José Daniel B. 592

Olortegui, Maribel Paredes 390

Omar, Rahila 289

Ombok, Maurice 301

Opryszko, Melissa C. 651

Ordaz-Farias, Alejandro 153

Oreagba, Ibrahim 14

Oremo, Jared 757

Orenstein, Walter 172

Oreste, María F. 578

Orozco, M. Marcela 872

Ortiz, Sylvia 285

Osilo, Emmanuel 736

Osman, Marwan 1203

Ostovar, Gholamabbas Amin 601

Ota, Martin O. C. 414

Otieno, Peter 301

Ouédraogo, Smaïla 292

Overgaard, Hans J. 727

\section{$\mathbf{P}$}

Pach, Alfred 144

Pacheco, Richard 937

Padilha, Amanda F. 937

Padilla-Docal, Barbara 230

Paes, Antonio Carlos 596

Pahissa, Albert 1196

Palmisano, Charles 986

Pan, Yi 172

Pandey, Kishor 227

Pandey, Krishna 222

Panella, Nicholas A. 116

Panosian Dunavan, Claire 203, 803

Panzera, Yanina 1146

Papadi, Bhavesh 1062
Paredes Olórtegui, Maribel 1109

Park, Mi-Yeoun 24

Parola, Philippe 397

Parraga, Isabel M. 433

Parsonnet, Julie 191

Parthasarathy, Sonaimuthu 883

Partida, Oswaldo 186

Pascalis, Hervé 329

Pasricha, Janet M. 20

Pasricha, Sant-Rayn 20

Patel, Jaymin C. 1116

Paterniti, Antonio D. 547

Patkar, Chinmay 89

Patterson, William 127

Pavio, Nicole 329

Paz, Carlos 583

Peacock, Sharon J. 411

Pedrosa-Corral, Irene 1003

Pellecer, Mariele J. 638

Peñaranda, Silvia 472

Pérez de Rosas, Alicia R. 893

Pérez-Ruiz, Mercedes 1003

Perrotti, Isabella Belletti Mutt 596

Perry, Diana 778

Perry, Sharon 191

Petri Jr., William A. 167

Petz, Lawrence N. 245

Phetsouvanh, Rattanaphone 932

Phommasone, Koukeo 932

Phong, Tran Vu 681

Phoumin, Phonlavanh 932

Pietz, Pamela J. 1152

Piffer, Michele 1070

Pinedo-Cancino, Viviana 559

Pinkerton, Relana 127

Pipatsatitpong, Duangnate 352

Pisarcik, Sarah E. 245

Plante, Kenneth S. 1170

Poché, David 778

Poché, Richard 778

Poirier, Philippe 1203

Pok, Kwoon-Yong 888

Polesky, Andrea 191

Poppert, Sven 198

Porco, Travis C. 138

Porphyre, Vincent 329

Portillo, Tobías 186

Postels, Douglas G. 315

Potchen, Michael J. 542

Pouladfar, Gholamreza 868

Pourabbas, Bahman 868

Poveda, Eva 789

Povoa, Marinete M. 1079

Powell, Jeffrey R. 897

Powers, Ann M. 116

Powers, Marilyn 929

Pozio, Edoardo 364

Pravia, Carlos 578

Prazeres da Costa, Clarissa 198

Prichard, Roger K. 1052

Psaroulaki, Anna 698

Puente, Sabino 789

Pukuta, Elisabeth 982

Pun, Sher Bahadur 227

Puri, Mahesh 144

Purkait, Bidyut 222

Putnak, Robert 73

Qadri, Firdausi 661

Qian, Men-Bao 1011 
Qiu, Jiamin 795

Querec, Troy 172

Quetz, Josiane 390

Quick, Robert E. 132, 267

Quipildor, Marcelo 578

Qureshi, Shahida 390

\section{$\mathbf{R}$}

Raby, Edward 481, 701

Racz, Paul 198

Radke, Elizabeth G. 908

Rainwater, Elizabeth 1011

Ramal Asayag, César 1109

Ramaseshan, Ganesh 481

Ramaswamy, Kalyanasundaram 883

Ramiro, Manuel 186

Ramos-Ligonio, Angel 566

Ranjan, Alok 222

Rao, Sowmya R. 376, 954

Raoult, Didier 339, 765, 1180

Rapley, Luke P. 490

Rasamoelina-Andriamanivo, Harentsoaniaina 329

Raso, José 1087

Rathbun, Stephen 426

Ratmanov, Pavel 339

Ratsimbasoa, Arsene 37

Rau, Gina 65

Ravinetto, Raffaella M. 608

Ray, Prabhati 1138

Reddy, Michael R. 897

Redfield, Robert R. 547

Rediske, Richard R. 862

Reeder, John C. 718

Reiche, Ana Silvia Gonzalez 108

Reis, Mitermayer G. 359

Reis, Renato B. 359

Renvoisé, Aurélie 765

Reyes, Miguel Ángel 186

Reynolds, Mary G. 982

Rezaee, Zahra 868

Ribeiro, Guilherme S. 359

Ribeiro, Márcio Garcia 596

Ribera, Esteve 1196

Richards, Allen L. 513

Richards, Frank O. 441

Ridl, Frances C. 897

Riera, Cristina 586

Rigg, Chystrie A. 572

Ritchie, Scott A. 65, 490

Rivard, Robert 929

Rivas, Pablo 789

Rivera, Esteban 319

Rizzo, Donna M. 630

Robinson, Sara 95

Rodas, Antonieta 630, 638

Roestenberg, Meta 5

Rogers, William O. 1116

Rojas, Liliana 186

Román, Karina 552

Romero-Vivas, Claudia M. E. 178

Rooban, Nirooshan 481

Rosenbaum, Laura 167

Rosenthal, Philip J. 289, 744

Rossi, Shannan L. 1170

Roucher, Clémentine 339

Roy, Somenath 828

Rubahika, Denis 855

Ruel, Theodore 744

Russo, Elizabeth 267

Ruth, Laird J. 757

Ryan, Edward T. 376, 661, 954

\section{$\mathbf{S}$}

Saavedra, Marlon 319

Sadumah, Ibrahim 132, 757

Saito, Mayuko 552

Sakai-Valente, Neusa Y. 236

Sala, Montserrat 914

Saldaña, Azael 572

Salomon, Martín 1207

Salvador, Fernando 1196

Samake, Sibiry 583

Sambo, Yohanna 441

Sampson-Johannes, Adam 1163

Sananikhom, Pany 1070

Sanbonmatsu-Gámez, Sara 1003

Sanchez, Juan Francisco 319

Sánchez-Vargas, Irma 260

Sandalakis, Vassilios 698

Sandoval, Carlos 1041

Santiago, Luis Manuel 472

Santos, Adolfo Carlos Barreto 596

Santos, Marina L. S. 325

Sardar, Abul Hasan 222

Sasaki, Mizuki 367

Sasaki, Toshinori 681

Satimai, Wichai 254

Sattabongkot, Jetsumon 1116

Sauerwein, Robert W. 5

Sawabe, Kyoko 681

Sawanyawisuth, Kittisak 461

Sawyer, Lynette 1163

Saxton-Shaw, Kali D. 103

Schieffelin, John S. 241

Schijman, Alejandro G. 872

Schlagenhauf, Patricia 397

Schlichting, Carl D. 1079

Schluter, W. William 464

Scholzen, Anja 5

Schultz, Lee 718

Schuster, Anthony L. 1116

Schwab, Kellogg J. 651

Schwartz, Eli 397

Schwenkenbecher, Jan M. 1052

Sciotti, Richard J. 216

Scott, Kerry 1109

Scott, Thomas W. 689

Seas, Carlos 1207

Sears, Stephen 95

Sedai, Tika Ram 464

Segura, Ferran 914

Seidman, Jessica 390

Seifert, Stephanie N. 689

Senn, Nicholas 718

Sevilla-Gonzalez, Farah K. 153

Seydel, Karl B. 542

Seymour, Robert L. 1170

Shah, Muhammad Ali 519

Shah, Rajesh 227

Shakely, Deler 289

Shakya, Geeta 464, 677

Shambare, Donald 645

Shao, Zhujun 923

Sharlow, Elizabeth R. 216

Shasha, Wang 770

Sheth, Anandi 267

Sheybani, Fereshte 469

Shields, Michael 607

Shin, E-Hyun 24

Shively, Kirk J. 95

Shrestha, Murari Man 464

Shrestha, Sanjaya 390

Shrestha, Sanjaya K. 464

Siba, Peter M. 718

Sibounheuang, Bountoy 932
Silk, Benjamin J. 132

Silva, Sonia L. 592

Sim, B. Kim Lee 5

Simpson, Andrew J. H. 411

Singh, Abhishek Pratap 1

Singh, Dharmendra 222

Singh, Shubhankar Kumar 222

Siripattanapipong, Suradej 352

Sisson, Andrew J. 862

Slotman, Michel A. 897

Smith, David W. 481

Smith, Leticia B. 95

Smith Jr., Robert P. 95

Smith, Tyler B. 908

Smits, Henk L. 552

Smucker, Joanne E. 572

Socarrás-Hernández, Carmen Aleida 230

Sokhna, Cheikh 339

Solari, Aldo 285

Song, Zhizhong 923

Soriano, Vincent 789

Sosa, Silvia M. 116

Sotir, Mark J. 376

Sovada, Marsha A. 1152

Specht, Katja 198

Speers, David J. 481

Speich, Benjamin 626

Sserwanga, Asadu 855

Ssewanyana, Isaac 747

Staedke, Sarah G. 1102

Staples, J. Erin 954

Steinhardt, Laura C. 855

Stevens, Lori 630

Stewart-Ibarra, Anna M. 971

Stoller, Nicole E. 138

Stroppa, María M. 1146

Suchdev, Parminder S. 757

Sulleiro, Elena 1196

Sullivan, Kevin M. 757

Sultana, Yasmin 1048

Sun, Wellington 73, 997

Sutherland, Genevieve 986

Suvari, Muhiddin 1032

Swope, Bethany N. 103

Sylla, Khadime 1124

\section{$\mathbf{T}$}

Taján, Juan 914

Takada, Nobuhiro 1217

Takagi, Masahiro 681

Tall, Adama 339

Tall, Koureishi 583

Tan, Cheong-Huat 888

Tan-ariya, Peerapan 352

Tantalean, Manuel E. 1212

Tappe, Dennis 198

Tappero, Jordan W. 736

Tauzie, Blessius 267

Taylor, Terrie E. 315, 542

Taylor, Viki G. 986

Tchuem Tchuenté, Louis-Albert 426

Teelen, Karina 5

Teirlinck, Anne C. 5

Temmam, Sarah 329

Tesh, Robert B. 455

Teuwen, Dirk 172

Thammakumpee, Kreeta 461

Thathaisong, Umaporn 352

Thiry, Dorothy 178

Thojaikong, Thaksinaporn 932

Thomas, Charles L. 433 
Thomas, Stephen J. 73

Thomsen, Thomas T. 536

Thong, Kwai Lin 704

Thriemer, Kamala 144

Tianyu, Guo 770

Tichit, Magali 37

Timiryasova, Tatyana M. 962

Tine, Roger 1124

Tinto, Halidou 608

Toledo, Andrea 285

Tomás, Elsa V. E. 536

Tomás-Pérez, Míriam 586

Tomashek, Kay M. 472, 997

Tong, Carlos 1130

Tong, Shilu 526

Tong, Zhaohui 923

Topno, Kamal Roshan 222

Torres-Aponte, Jomil M. 997

Toussaint, Jean-Francois 73

Trang, Bui Minh 681

Traore, Pierre 583

Trape, Jean-François 339

Travassos da Rosa, Amelia P. A. 455

Treviño, Ana 789

Treviño-Garza, Nancy 153

Trindade, Maria Ângela B. 236

Trongtokit, Yuwadee 254

Tsetsarkin, Konstantin A. 1163

Tsuda, Yoshio 681

Tucker, J. Allan 1062

Turab, Ali 390

Turell, Michael J. 245

Turmel, Jon P. 103

\section{$\mathbf{U}$}

Ubalee, Ratawan 1116

Udhayakumar, Venkatachalam 850

Ueda, Takanori 1217

Umaru, John 441

Umezawa, Eufrosina Setsu 559

Umhang, Gérald 795

Upreti, Senendra R. 677

Upreti, Shyam Raj 464

Ustun, Narmin 929

Utzinger, Jürg 626

\section{$\mathbf{V}$}

Vaca, Maritza 1041

Vaidyanathan, Rajeev 619

Valadez, Alicia 186

Valea, Innocent 608

Valencia, Braulio Mark 774

Valenzuela, Jesus G. 583

Valim, Clarissa 315

Van der Stuyft, Patrick 254

van der Ven, André J. A. M. 5

van Lieshout, Lisette 426, 1028

Vanlandingham, Dana L. 1163

Vanlerberghe, Veerle 254

Vardo-Zalik, Anne M. 29
Vasconcelos, Dewton de M. 236

Vázquez, Susana 506

Veland, Nicolas 774

Velmurugan, Soundarapandian 5

Vera, Hubert 319

Veracx, Aurélie 1180

Vergara, Fernanda 285

Vergne, Edgardo 997

Verma, Neena 222

Verma, Rakesh Bihari 222

Verweij, Jaco J. 1028

Vicuña, Yosselin 1041

Vijitsopa, Teerapon 461

Villalobos, Guiehdani 275

Villalpando, Carlos 578

Villar del Saz, Sara 1196

Vinetz, Joseph M. 184, 319, 552, 1130

Viscogliosi, Eric 1203

Vivar, Aldo 552

Voge, Natalia V. 260

von Seidlein, Lorenz 144

von Sonnenburg, Frank 397

Vongphayloth, Khamsing 1070

Vulcano, Luiz Carlos 596

Vulule, John 301

Vythilingam, Indra 888

\section{W}

Wadegu, Meshack 940

Wahlgren, Mats 733

Wald, Coridalia 572

Walker, David H. 937

Walker, Edward D. 301

Wampler, Peter J. 862

Wandera, Bonnie 1102

Wang, Chunmei 420

Wang, Guangfa 923

Wang, Ling 510

Wanga, Charles L. 608

Wanjala, Atsabina 344

Wanzira, Humphrey 736, 855

Wattleworth, Matthew 547

Watts, Matthew R. 1048

Wawrzyniack, Ivan 1203

Weaver, Scott C. 1170

Webber, Lori 95

Weil, Gary J. 1035

Weina, Peter J. 1138

Wen, Yan 918

Were, Vincent 132

Wesson, Dawn M. 986

West, Sheila K. 138

Whitehead, Susan 20

Wiedeman, Caleb 162

Wiegand, Ryan E. 855

Wierzba, Thomas 144

Wilkins, Kimberly 982

Wilkins, Patricia P. 1035

Williams, Craig 65

Williamson, John 301

Williams, Thomas N. 757

Wilson, L. Anthony 127

Wilson, Lawrence A. 986

Winch, Peter J. 1109
Witkowski, Benoit 37

Wongsrichanalai, Chansuda 1116

Wood, Zachary 167

Wren, Brendan W. 519

Wright, James H. 157

Wurapa, Eyako K. 940

$\mathbf{X}$

Xiaohong, Sun 770

Xiao, Shu-Yuan 455

Xie, Xu 923

Ximénez, Cecilia 186

Xing, Yan 918

$\mathrm{Xu}$, Jiabao 420

\section{$\mathbf{Y}$}

Yamamoto, Seigo 1217

Yamamoto, Taro 727

Yamwong, Sukit 461

Yan, Guiyun 29, 420

Yang, Henling 526

Yang, Tielong 923

Yang, YanFen 1209

Yap, Grace 1065

Yaregal, Zelalem 785

Yazdanbakhsh, Maria 1028

Yeka, Adoke 855

Yen, Nguyen Thi 681

Yingqun, Fu 770

Yong, Hou 770

Yoo, Dae-Hyun 24

Yori, Pablo Peñataro 390

Yu, Hao 510

Yu, Sun N. 138

Yu, Weiwei 526

Yu, Xue-Jie 510

Yu, Yang 770

Yuan, Lian-Chao 918

\section{$\mathbf{Z}$}

Zambrano, Jose Orlinder Nicolas 850

Zedar, Rebecca 962

Zeng, Guang 923

Zermeño, Valeria 186

Zeziulin, Oleksandr 1011

Zhang, Hongtao 923

Zhang, Peng 1138

Zhang, Shou-Feng 510

Zhang, Ying 918

Zhao, Hui 982

Zhong, Daibin 29

Zhou, Guofa 29

Zhou, Xiaohong 420

Zhou, Xiao-Nong 526

Zhou, Zhaoxia 138

Zuo, WeiWei 1209

Zuo, YangXian 1209 